\title{
Welche Patienten sind Kandidaten für die transnasale Insufflation?
}

Nilius $G$ et al. Predictors for treating obstructive sleep apnea with an open nasal

cannula system (transnasal insufflation). Chest 2010;137:521-528

\section{Hintergrund}

25-50\% aller Patienten mit obstruktiver Schlafapnoe (OSA) tolerieren die indizierte Therapie nicht. Die Hauptursache liegt in der gewöhnungsbedürftigen Maske und dem zuführenden Schlauchsystem, das erforderlich ist, um einen Überdruck in den Atemwegen zu erzeugen. Wünschenswert wäre deshalb eine vereinfachte Möglichkeit zur Druckerhöhung.

Mit der transnasalen Insufflation (TNI) von Luft gelingt es, den endexspiratorischen pharyngealen Druck geringfügig zu erhöhen. Dabei liefert ein Kompressor mit einer Flussrate von bis zu $20 \mathrm{l} / \mathrm{min}$ Luft, die dem Patienten über eine $\mathrm{Na}$ senkanüle zugeführt wird. Die Luft wird auf $30-33^{\circ} \mathrm{C}$ angewärmt und über einen Befeuchter auf $80 \%$ relative Luftfeuchtigkeit angefeuchtet. Die Atemwege werden durch die Nasenkanüle nicht komplett verschlossen. Man kann deshalb von einer „offenen CPAP-Methode“ sprechen.

\section{Methodik}

42 Patienten mit unterschiedlichen Schweregraden einer OSA wurden untersucht. Eine Apnoe wurde identifiziert, wenn der Atemfluss länger als zehn Sekunden nahe null lag, eine Hypopnoe, wenn der Atemfluss länger als zehn Sekunden um > 30\% und die $\mathrm{O}_{2-}$ Sättigung um > 3\% abfiel.

Als therapeutisch relevanter Erfolg wurde u.a. definiert, wenn der Respiratory Disturbance Index (RDI) initial über 10/h lag und um mindestens 50\% auf unter $10 / \mathrm{h}$ reduziert wurde.

Um zu eruieren, welche Kriterien vorliegen sollten, um erfolgreich therapieren zu können, wurde nach verschiedenen Schweregraden differenziert:

- Patienten mit Weckreaktionen, die überwiegend mit Atemanstrengung verbundenen waren (respiratory related arousals, RERA),

- Patienten mit überwiegend im REM-

Schlaf auftretenden Apnoen und $\mathrm{Hy}$ popnoen,

- Patienten mit > 50\%, > 66\% und $>90 \%$ Hypopnoen.

\section{Ergebnisse}

Der RDI der 42 Patienten (d.h. die Summe aller Apnoen, Hypopnoen und respiratorisch bedingten Weckreaktionen) lag bei 22,6 Ereignissen/h. Eine TNI führte zu einer geringen, signifikanten Reduktion

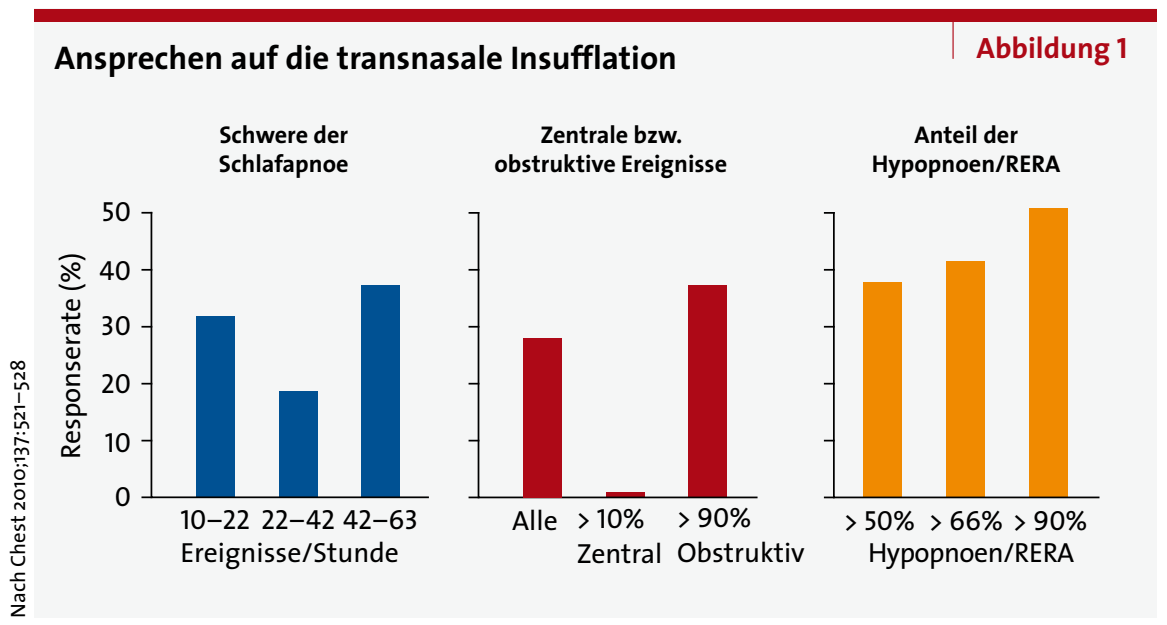

auf 17,2 Ereignisse/h. Klinisch akzeptabel war dieser Effekt bei $27 \%$ aller untersuchten Patienten. Die TNI führte zu einer Konversion von Apnoen zu Hypopnoen.

Das wichtigste Ergebnis war, dass die höchste Responserate bei Patienten mit überwiegend Hypopnen, RERA und REM-assoziierten Ereignissen zu erwarten ist (Abb. 1 rechts).

Patienten mit überwiegend Apnoen hatten eine geringe Responserate von $18 \%$. Wenig erfolgreich war die TNI außerdem bei Patienten mit > 10\% zentralen Apnoen (Abb. 1 Mitte). Anthropometrische Daten wie Geschlecht, Alter und Body-Mass-Index hatten keinen Einfluss auf das Ergebnis, ebenso wenig der Apnoe-Hypopnoe-Index und die Höhe des titrierten CPAP-Werts.

\section{Schlussfolgerungen}

Durch eine TNI mit Flussraten bis zu $20 \mathrm{l} / \mathrm{min}$ wird der Druck in den oberen Atemwegen um etwa $2-3 \mathrm{cmH}_{2} \mathrm{O}$ erhöht. Schon bei dieser geringen Druckerhöhung steigt der inspiratorische Fluss um etwa $100 \mathrm{ml} / \mathrm{s}$ an. Dieser Druck scheint auszureichen, um die Atmung mit Hypopnoen, bei denen wir eine Flussrate von etwa $150-200 \mathrm{ml} / \mathrm{s}$ finden, zu stabilisieren. Der Effekt war besonders wirksam im REM-Schlaf, der im Vergleich zum Nicht-REM-Schlaf mit noch stärker ausgeprägter Hypotonie der Muskulatur der oberen Atemwege einhergeht.

\section{Kommentar}

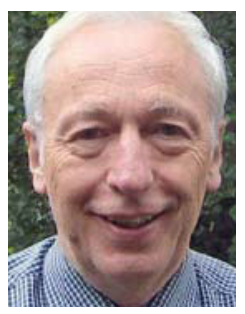

Prof. Dr. med. Karl-Heinz Rühle, Hagen

Bei bestimmten Untergruppen wie z.B. Kindern oder Erwachsenen mit überwiegend Hypopnoen und Patienten mit Upper-Airway-Resistance-Syndrom könnte die TNI als Alternative zur Atmung mit nasalem positivem Überdruck (nCPAP) in Erwägung gezogen werden. Allerdings sind noch Untersuchungen zur Langzeitanwendung erforderlich, um die Indikation und den klinischen Nutzen genauer abzusichern. 